\title{
An Efficient Numerical Method for 3D Viscous Ship Hydrodynamics with Free-Surface Gravity Waves
}

\author{
Mervyn Lewis and Barry Koren \\ CWI, P.O. Box 94079, 1090 GB Amsterdam, The Netherlands \\ [Mervyn.Lewis, Barry. Koren] @cwi.nl
}

Summary. A new numerical method for water flows with free-surface gravity waves is investigated. The method is first analyzed with respect to the existence of steady free-surface waves, and the dispersion properties of these waves. Next, the method is used to compute the free water surface generated by a standard ship hull.

\section{Introduction}

Most of the current numerical methods for solving steady, viscous free-surface flows in ship hydrodynamics employ a time-dependent formulation and integrate until a steady state is reached. This approach typically displays the defect of high computational costs due to persistent transient behaviour of the long gravity waves. The attenuation of these waves behaves like $\mathcal{O}\left(t^{(1-d) / 2}\right)$ in $\mathbb{R}^{d}$. To reduce the computational effort of solving free-surface Navier-Stokes flow, an efficient iterative method, employing a stationary formulation of the problem, has been introduced for $2 \mathrm{D}$ in [1] and has been extended to 3D in [3]. The method relies on a novel formulation of the free-surface flow problem, involving the so-called quasi free-surface condition (QFSC):

$$
\boldsymbol{u} \cdot \nabla \varphi-\mathrm{Fr}^{-2} \boldsymbol{u} \cdot \boldsymbol{e}_{z}=0
$$

where $\boldsymbol{u}$ is the velocity vector, $\varphi$ the hydrodynamic pressure:

$$
\varphi=p+\operatorname{Fr}^{-2} z,
$$

Fr the Froude number and $\boldsymbol{e}_{z}$ the unit vector in vertical direction, positive upward. Note that the boundary condition (1a), see [1] for a derivation, is nonlinear. So far, this method has not yet been applied to a real 3D ship-hydrodynamics problem. In the present paper we will do so. Moreover, a Fourier analysis will be made of a semi-discrete generic model problem representing our discrete, 3D Navier-Stokes flow problem. In the Navier-Stokes equations considered, streamwise diffusion, i.e., diffusion in $x$-direction, is neglected. This simplification turns out to have far-reaching consequences for the well-posedness of the continuous and semidiscrete initial boundary-value problem.

\section{Fourier analysis of semi-discrete problem}

As mentioned above, the aim is to compute the complete stationary flow field, i.e., the shape of the free surface and the underlying viscous flow field, generated by a 
ship. We start by analyzing the wave pattern by performing a Fourier analysis for a $2 \mathrm{D}$, uniform horizontal flow in infinitely deep water. The analysis uses classical perturbation methods, linearization and Fourier techniques. We assume perturbation expansions in powers of $\epsilon, \epsilon \ll 1$, of the form

$$
\begin{gathered}
\boldsymbol{q}(\boldsymbol{x}, t ; \epsilon)=\boldsymbol{q}_{0}+\epsilon \boldsymbol{q}_{1}(\boldsymbol{x}, t)+\mathcal{O}\left(\epsilon^{2}\right), \\
\eta(x, t ; \epsilon)=\eta_{0}+\epsilon \eta_{1}(x, t)+\mathcal{O}\left(\epsilon^{2}\right),
\end{gathered}
$$

where the solution vector is represented by $\boldsymbol{q}=(\boldsymbol{u}(\boldsymbol{x}, t), \varphi(\boldsymbol{x}, t))^{T}$ and where $\eta=\eta(x, t ; \epsilon)$ denotes the water height. The generating solution, denoted by $\boldsymbol{q}_{0}=(\boldsymbol{U}, \Phi)^{T}$ and $\eta_{0}$, consists of the unperturbed flow velocity $\boldsymbol{U}=(U, 0)^{T}(U=$ constant), the unperturbed hydrodynamic pressure $\Phi=0$ and the unperturbed water height $\eta_{0}=0$. Our spatial domain $\Omega$ is defined as

$$
\Omega=\left\{\boldsymbol{x} \in \mathbb{R}^{2}: x \in(-\pi, \pi), z \in(\eta,-\infty)\right\} .
$$

Since we are considering the flow in infinitely deep water, in $z$-direction the domain $\Omega$ is only bounded by the free surface. We assume that

$$
\lim _{z \downarrow-\infty} \boldsymbol{q}_{1}(\boldsymbol{x}, t)=\mathbf{0} .
$$

Further, we take

$$
\boldsymbol{q}(-\pi, z)=\boldsymbol{q}(\pi, z)
$$

i.e., periodicity of the solution in $x$-direction. To study the combined effect of the different truncation errors on the numerical approximation of the free-surface flow, we consider the system of modified equations resulting from a semi-discretization of the reduced Navier-Stokes equations in $\mathbb{R}^{2}$, on a uniform Cartesian grid with mesh width $h$. This system reads, neglecting the $\mathcal{O}\left(h^{4}\right)$ terms,

$$
\begin{aligned}
u_{t}+U u_{x}+\varphi_{x}-\operatorname{Re}^{-1} u_{z z} & =U \frac{h^{2}}{3} u_{x x x}+\frac{h^{3}}{12} \varphi_{x x x x}+\operatorname{Re}^{-1} \frac{h^{2}}{12} u_{z z z z} \\
w_{t}+U w_{x}+\varphi_{z}-\operatorname{Re}^{-1} w_{z z} & =U \frac{h^{2}}{3} w_{x x x}+\frac{h^{3}}{12} \varphi_{z z z z}+\operatorname{Re}^{-1} \frac{h^{2}}{12} w_{z z z z} \\
u_{x}+w_{z} & =\frac{h^{3}}{3} u_{x x x}+\frac{h^{3}}{12} w_{z z z z}
\end{aligned}
$$

\subsection{Second-order accurate upwind discretization of QFSC}

On the free-surface boundary $z=\eta_{0}$, the modified quasi-free surface condition resulting from an $\mathcal{O}\left(h^{2}\right)$ upwind-biased spatial discretization is imposed, i.e.,

$$
\varphi_{t}+U \varphi_{x}-\mathrm{Fr}^{-2} w=U \frac{h^{2}}{3} \varphi_{x x x}+\mathcal{O}\left(h^{3}\right)
$$

is imposed. To construct a Fourier representation of the solution $\boldsymbol{q}_{1}(\boldsymbol{x}, t)$, consider the following isolated mode:

$$
\boldsymbol{q}_{1}(\boldsymbol{x}, t)=\hat{\boldsymbol{q}}(k, s, \omega) e^{i k x+s z-i \omega t},
$$

where $k, s \in \mathbb{R}$ are the wave numbers in $x$ - and $z$-direction, respectively, and where $\omega \in \mathbb{R}$ is the radial frequency. Substitution of (7) into (5), neglecting the higher than $\mathcal{O}\left(h^{2}\right)$ terms, yields 


$$
\begin{gathered}
\hat{\boldsymbol{L}}_{h}(k, s, \omega) \hat{\boldsymbol{q}} e^{i k x+s z-i \omega t}=\mathbf{0} \\
\hat{\boldsymbol{L}}_{h}(k, s, \omega)=\left(\begin{array}{ccc}
\hat{H}_{h}(k, s, \omega) & 0 & \hat{G}_{x, h} \\
0 & \hat{H}_{h}(k, s, \omega) & \hat{G}_{z, h} \\
\hat{D}_{x, h} & \hat{D}_{z, h} & 0
\end{array}\right),
\end{gathered}
$$

with

$$
\hat{H}_{h}(k, s, \omega)=-i \omega+U i k\left(1+\frac{h^{2} k^{2}}{3}\right)-\operatorname{Re}^{-1} s^{2}\left(1+\frac{h^{2} s^{2}}{12}\right)
$$

the semi-discrete convection-diffusion operator, and with

$$
\left(\begin{array}{c}
\hat{G}_{x, h} \\
\hat{G}_{z, h}
\end{array}\right)=\left(\begin{array}{c}
i k \\
s
\end{array}\right), \quad\left(\begin{array}{c}
\hat{D}_{x, h} \\
\hat{D}_{z, h}
\end{array}\right)=\left(\begin{array}{c}
i k\left(1+\frac{h^{2} k^{2}}{3}\right) \\
s
\end{array}\right)
$$

the discrete pressure gradient operator and divergence operator, respectively. A nontrivial solution of (8a) exists only if

$$
\operatorname{det}\left(\hat{\boldsymbol{L}}_{h}\right)=0
$$

which results in

$$
s=k\left(1+\frac{h^{2} k^{2}}{6}\right)
$$

Here we have used the fact that for $z \downarrow-\infty$ the solution perturbations are zero. So, the corresponding solution of the system of modified equations is

$$
\boldsymbol{q}_{1}(\boldsymbol{x}, t)=\left(\begin{array}{c}
\hat{G}_{x, h} \\
\hat{G}_{z, h} \\
-\hat{H}_{h}(k, s(k), \omega)
\end{array}\right) e^{i k x+k\left(1+\frac{h^{2} k^{2}}{6}\right) z-i \omega t}
$$

The dispersion relation for the modified problem follows by substitution of this solution into (6) and solving for $\omega$. The roots $\omega_{1,2}$ can be calculated explicitly, giving

$$
\begin{gathered}
\omega_{1,2}=U \tilde{k} \pm \frac{1}{2} \sqrt{4 \mathrm{Fr}^{-2} s-\mathrm{Re}^{-2} \tilde{s}^{4}}+i \frac{1}{2} \mathrm{Re}^{-1} \tilde{s}^{2}, \\
\tilde{k} \equiv k\left(1+\frac{h^{2} k^{2}}{3}\right), \quad \tilde{s}^{2} \equiv s^{2}\left(1+\frac{h^{2} s^{2}}{12}\right) .
\end{gathered}
$$

Note that the presence in (12a) of the positive imaginary term $i \operatorname{Re}^{-1} \tilde{s}^{2}$ gives rise to exponentially growing solutions. Hence, here steady free-surface waves cannot exist for finite Reynolds number. It can be shown that this is not due to the discretization but due to the continuous formulation of the free-surface flow problem. This suggests that this formulation of the free-surface flow problem is formally ill-posed. However, the measure of ill-posedness is small for large Re.

In the inviscid limit a stationary wave is found. For $\operatorname{Re} \uparrow \infty$ and $h \downarrow 0, \omega_{1,2}=0$ in $(12 \mathrm{a})$ yields

$$
U^{2} k\left(1+\frac{h^{2} k^{2}}{2}\right)=\mathrm{Fr}^{-2}
$$

This relation implies that for fixed $U$ and Fr, $k$ decreases with increasing $h$. I.e., a coarser mesh results in a larger length of the free-surface wave. Solving the previous expression for $k$, assuming a solution of the form $k=(U \mathrm{Fr})^{-2}(1+\epsilon), \epsilon \ll 1$, yields, neglecting higher-order terms of $\epsilon$, 


$$
k=\frac{1}{(U \mathrm{Fr})^{2}}\left(1-\frac{\frac{1}{2} h^{2}}{(U \mathrm{Fr})^{4}}\right)
$$

We investigate the correctness of (14) for the numerical results depicted in Figure 1 . Here we have applied three grids with in $x$-direction the mesh sizes $\frac{h}{2}, h$, and $2 h$.

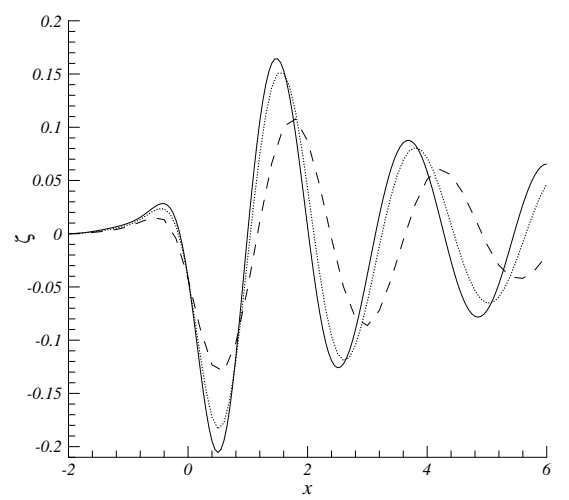

Fig. 1. Wave elevation in some plane $y=$ constant for a 3D test case from Chapter 2 of [2], with $U=1$ and $\mathrm{Fr}=0.6$.

For $U=1, \mathrm{Fr}=0.6$ and through the wavelength relation $k=\frac{2 \pi}{\lambda}$, according to (14) it should then hold: $\lambda_{\frac{h}{2}}=2.27, \lambda_{h}=2.31, \lambda_{2 h}=2.48$, with $\lambda_{h=0}=2.26$. Detailed observation of the results shown in Figure 1 shows that the above, analytically found wavelengths are quite accurate, thus proving that the Fourier analysis is not only of qualitative value but even of quantitative value.

\subsection{First-order accurate upwind discretization of QFSC}

In this section we analyze the effect of an $\mathcal{O}(h)$-upwind discretization of the quasi free-surface condition. The modified equation belonging to the $\mathcal{O}(h)$-upwind discretization is

$$
\varphi_{t}+U \varphi_{x}-\mathrm{Fr}^{-2} w=U \frac{h}{2} \varphi_{x x}+\mathcal{O}\left(h^{2}\right)
$$

Applying the Fourier transform to the previous expression and substitution of the solution (11) into the Fourier-transformed quasi free-surface condition (15) yields again a dispersion relation. The solution of this quadratic equation for $\omega$, neglecting the $\mathcal{O}\left(h^{2}\right)$ term, results in the following roots

$$
\omega_{1,2}=\frac{2 U k+i\left(\operatorname{Re}^{-1} s^{2}-\frac{h}{2} U k^{2}\right) \pm \sqrt{4 \mathrm{Fr}^{-2} s-\left(\operatorname{Re}^{-1} s^{2}+\frac{h}{2} U k^{2}\right)^{2}}}{2} .
$$

Note that now, as real part of the solution, we have (at least) $e^{\frac{1}{2}\left(\operatorname{Re}^{-1}-\frac{h}{2} U\right) k^{2} t}$, where we have again neglected the $\mathcal{O}\left(h^{2}\right)$ terms. Whereas for both the exact equations and the $\mathcal{O}\left(h^{2}\right)$ modified equations, the time behaviour is always exponentially 
growing, with the $\mathcal{O}(h)$ upwind discretization of the QFSC, it can be exponentially decreasing, i.e., it can lead to a steady wave. From (16), it appears that a steady wave is obtained if

$$
U \operatorname{Re} h>2 .
$$

Considering the case $U=1, \ell=1$, where $\ell$ is the reference length, this means that for mesh Reynolds numbers Reh $>2$ we may obtain steady waves due to the numerical diffusion introduced by the $\mathcal{O}(h)$ upwind discretization of the pressure derivative in the QFSC. In our practical computations, with $h \div \operatorname{Re}^{-\frac{1}{2}}$, this condition is easily satisfied.

\section{Numerical results}

The free-surface algorithm is now applied to a standard test case originating from ship hydrodynamics: the computation of the complete flow field generated by a Series 60 hull at $\mathrm{Fr}=0.316$ and $\operatorname{Re}=10^{6}$.

The computational domain for this test case contains $353 \times 69 \times 45$ grid points and for the initial $\Gamma_{\mathrm{FS}}$ we take $z=0$. Here, in the solution process, (1a) is treated using Newton's method and $\nabla \varphi$ is discretized employing the $\mathcal{O}(h)$-upwind scheme. The solution method corresponds to the modified algorithm as described in Chapter 2 of [2]. Two types of solutions are computed: $(i)$ solutions belonging to a linearization of the free-surface flow, the so-called 'uniform-flow' linearization (described in Chapter 4 of [2]) and (ii) solutions of the fully nonlinear free-surface flow problem. A comparison of the computed results with the experimental data, obtained from [4], is shown in Figure 2.
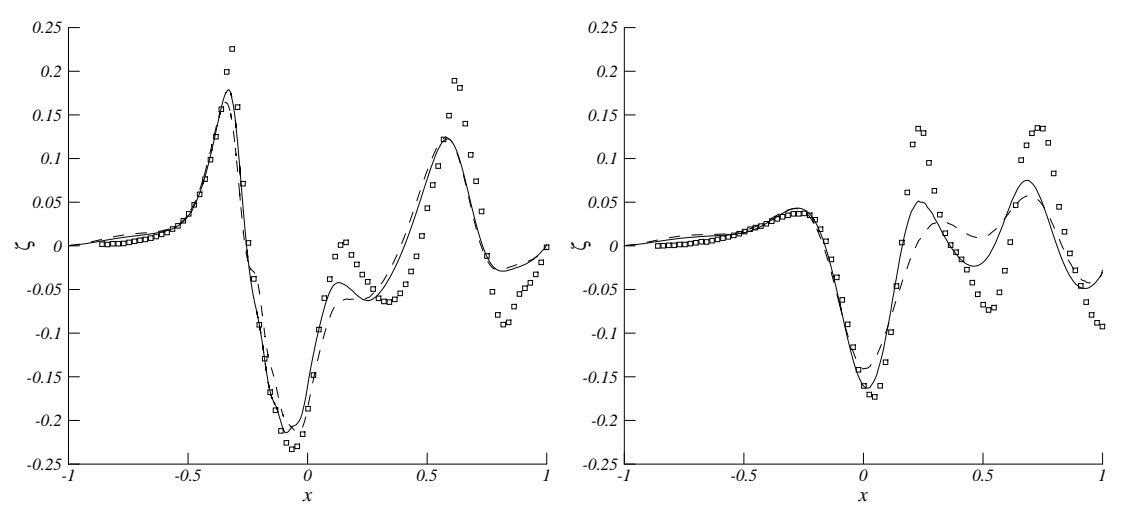

Fig. 2. Comparison of the computed longitudinal wave cuts, obtained from the nonlinear method (solid), the 'uniform-flow' linearization method (dashed), with experimental results (markers). Left: $y=0.0755 \ell$, right: $y=0.2067 \ell, y=0$ corresponds to the plane of symmetry. 


\section{Conclusions}

A Fourier-type analysis shows that for finite Reynolds number, steady free-surface waves cannot exist for our reduced Navier-Stokes equations, in combination with the quasi free-surface condition, neither in the continuous case nor in the fully second-order accurate discrete case. In agreement with our computational findings, the Fourier analysis also shows that for the reduced Navier-Stokes equations steady waves can exist when discretizing the quasi free-surface condition at first-order accuracy, under the constraint that the mesh Reynolds number does not exceed 2.

We have applied our iterative free-surface method to a real 3D ship hydrodynamics problem. The computed results reveal that the new formulation of the free-surface flow problem yields the correct wave physics and offers a fast convergence towards the nonlinear solution.

Acknowledgement: This work was supported by the Dutch Technology Foundation STW under grant CWI.4883.

\section{References}

1. E.H. van Brummelen, H.C. Raven, B. Koren: J. Comput. Phys 174, 120 (2001)

2. M.R. Lewis: Numerical methods for water flows with free-surface gravity waves. PhD Thesis, Delft University of Technology, Delft (2004)

3. M.R. Lewis, B. Koren, H.C. Raven: Computation of 3D steady Navier-Stokes flow with free-surface gravity waves. In: Proceedings of Computational Fluid Dynamics 2002, ed by S. Armfield, P. Morgan and K. Srinivas (Springer, Berlin 2003) pp 100-105

4. Y. Toda, F. Stern, J. Longo: Mean-flow measurements in the boundary layer and wake field of a Series $60 c_{b}=.6$ ship model for Froude numbers .16 and .316. Report 352, Iowa Institute of Hydraulic Research, Iowa City (1991) 